\title{
L'affirmation des multinationales de l'agroalimentaire des pays émergents. Le cas des firmes brésiliennes de la viande
}

\section{Cécile Fèvre et Thierry Pouch}

\section{OpenEdition}

\section{Journals}

Édition électronique

URL : http://journals.openedition.org/economierurale/3916

DOI : $10.4000 /$ economierurale.3916

ISSN : 2105-2581

Éditeur

Société Française d'Économie Rurale (SFER)

Édition imprimée

Date de publication : 15 mars 2013

Pagination : 85-98

ISSN : 0013-0559

\section{Référence électronique}

Cécile Fèvre et Thierry Pouch, «L'affirmation des multinationales de l'agroalimentaire des pays émergents. Le cas des firmes brésiliennes de la viande », Économie rurale [En ligne], 334 | mars-avril 2013, mis en ligne le 15 mars 2015, consulté le 20 avril 2019. URL : http://journals.openedition.org/ economierurale/3916 ; DOI : 10.4000/economierurale.3916 


\title{
FAITS ET CHIFFRES
}

\section{L'affirmation des multinationales de l'agroalimentaire des pays émergents Le cas des firmes brésiliennes de la viande}

\author{
Cécile FÈVRE • Direction économie des agricultures et des territoires \\ Assemblée Permanente des Chambres d'agriculture, Paris \\ Thierry POUCH • Direction économie des agricultures et des territoires \\ Assemblée Permanente des Chambres d'agriculture, Paris \\ Laboratoire Économie Gestion Agroressources Durabilité (REGARDS) \\ Université de Reims Champagne Ardenne, Reims
}

La montée en puissance du secteur agroalimentaire brésilien s'enclenche dès la fin de la décennie 1990. Elle prend la forme notamment d'une très forte croissance des exportations, favorisée par différentes variables macroéconomiques. Dans la période plus récente, ce sont les firmes multinationales (FMN) de l'agroalimentaire qui ont amplifié l'affirmation des produits brésiliens sur les marchés mondiaux. L'insertion du Brésil dans la mondialisation passe aussi par les stratégies d'implantation déployées par les firmes de taille mondiale. Cet article suggère d'en expliquer les raisons.

D ans le champ de l'économie internationale, un débat s'est très vite engagé dès le début du $\mathrm{XX}^{\mathrm{e}}$ siècle pour savoir si le fondement de la spécialisation restait, ou non, l'État-nation. L'évolution même de l'économie mondiale a montré que plusieurs acteurs contribuaient désormais à la construction de la spécialisation internationale (Kébabdjian, 1994 ; Rainelli, 2003) $)^{1}$. Il y eut d'abord l'approche centrée sur les États-nations. Il s'agissait d'espaces nationaux d'où les facteurs de production pouvaient se déployer et déterminer - à partir du jeu des avantages comparatifs relatifs (différentiels de productivités du travail chez Ricardo, dotations factorielles chez Heckscher-Ohlin-Samuelson) - le profil de la spécialisation internationale de chacun des participants à l'échange de biens et de services ${ }^{2}$.

$\mathrm{Si}$ le rôle des dotations factorielles demeure, celui des firmes multinationales

1. Kébabdjian G. (1994). L'économie mondiale. Enjeux nouveaux, nouvelles théories. Paris, Seuil ; Rainelli M. (2003). La nouvelle théorie du commerce international. Paris, La Découverte, coll. « Repères ».

2.Principe de l'échange de différences. dans la détermination de la spécialisation des économies est devenu important. L'accélération des Investissements directs à l'étranger (IDE), à partir du début des années 1960, a constitué en effet un bouleversement non seulement dans la dynamique de la mondialisation elle-même, mais aussi dans la manière d'appréhender statistiquement cette mondialisation. L'importance prise par les IDE réalisés par des firmes ayant défini leurs stratégies de conquête des marchés en jouant sur des critères de différenciation des territoires d'accueil, s'est généralisée à l'ensemble des secteurs d'activités économiques, de l'industrie à la finance en passant par les services. Concernant l'industrie, on sait que les firmes multinationales de l'agroalimentaire ont été depuis trente ans particulièrement dynamiques en matière d'IDE. Les flux de capitaux sont toutefois longtemps demeurés Nord-Nord, expression signifiant que la majorité des IDE étaient effectués par des firmes situées essentiellement dans les pays industrialisés. Ce n'est que récemment que les économies émergentes se sont insérées dans la hiérarchie des firmes réalisant des 
IDE. Ainsi, les flux d'IDE deviennent progressivement Sud-Sud ou même Sud-Nord, confirmant ainsi le phénomène - certes encore embryonnaire mais bien réel - de mutation de la hiérarchie de l'économie

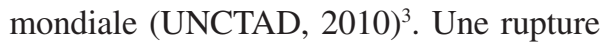
s'est introduite dans le leadership exercé antérieurement par les firmes nord-américaines et ouest-européennes sur le système productif mondial. L'agroalimentaire en forme une illustration récente. En opérant sur plusieurs continents, les firmes multinationales originaires des pays émergents participent du même coup à la structuration/intégration de l'espace productif mondial. Pour ce qui est du Brésil, dont il sera question dans cet article, l'internationalisation du capital amplifie le processus de création de richesses au-delà de l'espace productif national d'origine.

Pour illustrer cette nouvelle phase de la mondialisation du capitalisme et plus précisément de l'internationalisation du capital agroalimentaire, le cas des firmes brésiliennes de la viande a été retenu. Le Brésil s'est hissé, en l'espace d'une quinzaine d'années au rang de grande puissance productrice et exportatrice de produits agricoles et alimentaires ${ }^{4}$. En 2010, le Brésil s'est classé au troisième rang des exportateurs mondiaux de produits agroalimentaires, se positionnant derrière l'Union européenne à 27 (UE) et les États-Unis, mais devant l'Argentine. La présence des productions et des exportations brésiliennes sur les marchés mondiaux s'est étendue aux firmes de la transformation, lesquelles ont atteint des tailles suffisantes pour

3. UNCTAD (2010). World Investment Report. New York \& Geneva, United Nations.

4. Le Brésil était déjà une grande puissance agricole dès la seconde moitié du XIX ${ }^{\mathrm{e}}$ siècle. Mais sa spécialisation internationale dans ces secteurs reposait davantage sur des produits qualifiés d'exotiques (cacao, café, agrumes). Ses exportations agroalimentaires sont aujourd'hui beaucoup plus diversifiées, et concernent des produits typiques des climats tempérés. s'implanter à l'étranger et pour absorber - ou encore fusionner - certains de leurs concurrents.

Dans cet article, nous clarifierons d'abord par quelques éléments chiffrés la dynamique exportatrice du Brésil dans ce secteur de l'agroalimentaire. Elle forme en effet un premier axe à partir duquel s'est enclenchée la puissance du secteur agroalimentaire brésilien. Mais afin de contourner certaines des contraintes auxquelles se heurtent les exportations, allant de restrictions aux échanges - droits de douane ou barrières sanitaires - aux différentiels de taux de change, certaines firmes ont développé des stratégies d'implantation sur les marchés à travers des IDE. Nous proposons ensuite un éclairage sur la croissance des firmes multinationales du Brésil. Ces firmes sont parfois qualifiées de Global Players car elles définissent leur stratégie d'investissement, de production et de conquête de la demande sur l'intégralité de l'espace économique mondial. Cette stratégie vise à accentuer le processus de mise en valeur du capital, et tout particulièrement dans le domaine des viandes, avec pour conséquence un phénomène de rattrapage exercé sur les firmes américaines et européennes dont la transformation des viandes constitue le métier de base. Cet éclairage consistera d'une part en une approche quantifiée des IDE réalisés par les firmes brésiliennes, et d'autre part en une tentative de recensement des facteurs de la multinationalisation de ces mêmes firmes.

\section{L'exportation comme premier axe de l'essor brésilien sur les marchés mondiaux}

Jusqu'aux années 1990, l'UE et les ÉtatsUnis avaient dominé les marchés des produits agricoles et alimentaires. Ensuite, en raison d'une expansion spatiale de l'agriculture et de l'organisation productive du complexe agro-industriel - laquelle repose 
FAITS ET CHIFFRES

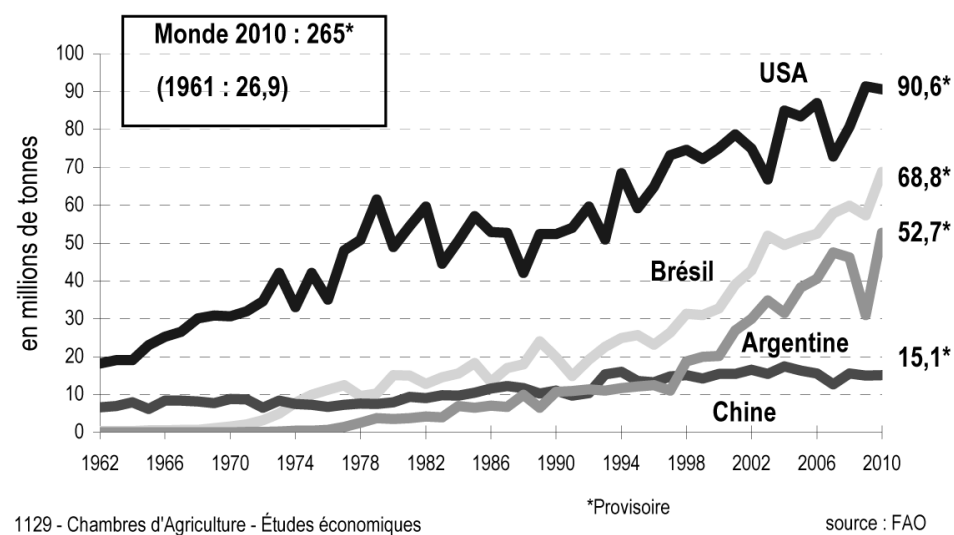

essentiellement sur une intégration verticale -, le Brésil s'affirme comme l'une des principales puissances agricoles dans l'économie mondiale (Dalla Costa, 2000 ; Bertrand, 2005) ${ }^{5}$. Très rapidement, cette économie se hisse au rang de principal producteur de soja, de viandes (bovins et volailles), de jus d'orange, de sucre, de café, mais aussi de biocarburants (éthanol à base de sucre de canne)... Ces productions vont faire l'objet d'une croissance fulgurante des exportations vers le reste du monde, le Brésil parvenant à se classer dans les trois premiers exportateurs mondiaux de produits agricoles et alimentaires (graphiques $1,2,3$, pour ne retenir que quelques exemples). En 2010, les exportations brésiliennes agroalimentaires ont représenté près de $34 \%$ des exportations totales du pays selon les données issues de Comptes harmonisés sur les échanges et l'économie mondiale du CÉPII (CHELEM), l'une des

5. Dalla Costa A. (2000). L'aviculture brésilienne face à la globalisation de l'économie. Cahiers $d u$ Brésil contemporain, $\mathrm{n}^{\circ}$ 41/42, p. 257-278 ; Bertrand J.-P. (2005). Politique agricole, stabilisation macroéconomique et politique commerciale au Brésil (1994-2004). In Rainelli P. (éd.), Les politiques agricoles sont-elles condamnées par la mondialisation ?, Louvain-la-Neuve, AcademiaBruylant, p. 55-91. parts les plus élevées dans la catégorie des pays émergents avec l'Argentine.

Par la suite, l'évolution du commerce extérieur agroalimentaire brésilien est nettement bien orientée, l'excédent retiré des échanges étant structurellement élevé (graphique 4). Cette nouvelle configuration de l'économie mondiale agricole, que l'on pourrait qualifier de multipolaire, s'est traduite en réalité par une montée des tensions, dont les blocages des négociations à l'OMC constituent une illustration tangible. Il faut entendre par là qu'un conflit commercial est en cours depuis une dizaine d'années, autour de la question de l'ouverture des marchés européen et américain aux produits agricole et alimentaire brésiliens, l'objectif du Brésil étant de faire valoir auprès de - plus exactement contre ses concurrents ses avantages comparatifs dans l'agroalimentaire (Pouch, 2010) ${ }^{6}$.

La stratégie du Brésil est de conquérir de plus en plus de parts de marché, de s'accaparer une rente à l'exportation, d'écouler la production, et donc de rentabiliser les capitaux investis dans le complexe agroalimentaire. Par ailleurs, la demande mondiale de

6. Pouch T. (2010). La guerre des terres. Stratégies agricoles et mondialisation. Paris, Choiseul. 


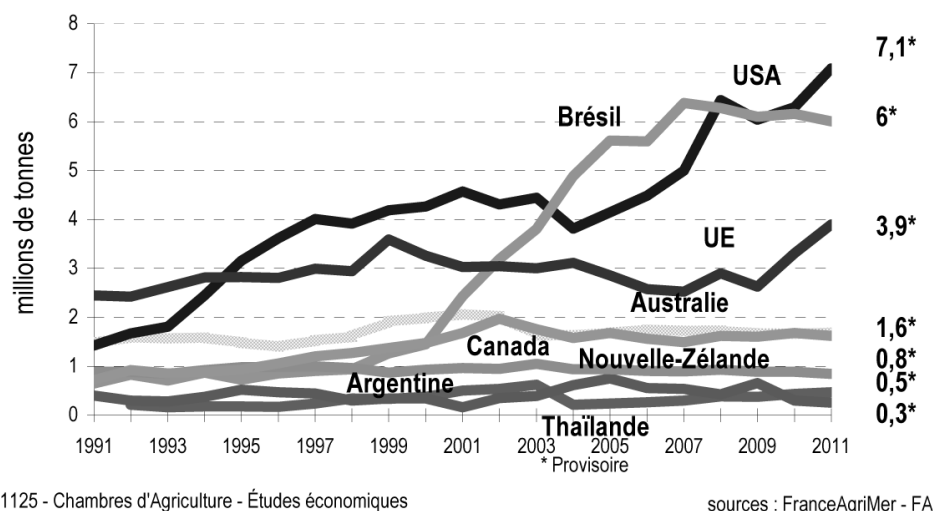

produits agricoles s'inscrit sur une dynamique ascendante, en raison notamment de l'accroissement de la demande chinoise. Au regard de ses productions animales destinées à répondre à une augmentation de la demande domestique imputable à l'élévation des niveaux de vie, la Chine importe massivement - elle absorbe plus de $50 \%$ des importations mondiales de soja depuis plusieurs années - du soja en provenance du Brésil (graphique 5). Les importations chinoises de graines oléagineuses ont de ce fait rattrapé celles réalisées par l'UE à 27. La dynamique de la demande mondiale de produits carnés - la FAO ayant indiqué que la production de viandes devrait doubler d'ici à 2050, hausse appelant à un surcroît de productions végétales, et de grains notamment - constitue pour le Brésil et son agro-industrie un véritable challenge.

L'autre dimension importante inhérente à la progression des exportations brésiliennes sur les marchés mondiaux tient au rattrapage fulgurant qu'a opéré ce pays vis-à-vis des États-Unis et de la France. La domination exercée par les États-Unis sur le marché des viandes n'est plus que relative, le Brésil faisant désormais jeu égal en ce domaine, et plus spécifiquement dans celui de la viande de volailles. C'est précisément sur ce terrain que la France et l'Union européenne ont dû affronter la concurrence brésilienne. La forte progression des viandes de volailles brésiliennes s'est faite d'ailleurs au détriment de leurs concurrents français et européens, lesquels ont été progressivement distancés du marché mondial. La part de l'UE dans les échanges mondiaux de viande de volailles est ainsi passée de $20 \%$ en 1994 à un peu moins de $10 \%$ en 2011, la part du Brésil évoluant quant à elle de 14 à $34 \%$, celle des États-Unis diminuant de 41 à $32 \% 7$. Le Brésil, qui entend jouer un rôle de premier ordre dans le développement de relations économiques Sud-Sud, s'est orienté vers les marchés des pays du Proche et du Moyen-Orient. Un véritable « corridor alimentaire » a été créé entre le Brésil et les pays composant le Proche et le Moyen-Orient (Abis, Nardonne, 2009) ${ }^{8}$. Exprimées en dollars courants, les exportations brésiliennes de viandes et autres préparations à base de viandes à destination des pays du Moyen-Orient non OPEP

7. Sources : FAO, USDA et Commission européenne. 8. Abis S., Nardone J. (2009). Le Brésil, future ferme du monde arabe ? Futuribles, $\mathrm{n}^{\circ} 356$, octobre, p. 13-29. 
FAITS ET CHIFFRES

(Organisation des Pays Exportateurs de Pétrole) sont passées de 9 à 330 milliards de dollars (CHELEM), soit une progression de $3560 \%$ entre 1990 et 2009.

\section{Une concurrence exacerbée à l'échelle mondiale}

La montée en puissance de pays émergents comme le Brésil ne fait qu'illustrer le fractionnement de l'économie mondiale agricole. On ne peut dès lors raisonner à partir d'une économie mondiale sans frontières, mais seulement en fonction d'une rivalité permanente à laquelle se livrent des unités nationales ou régionales séparées, non seulement du fait de leurs intérêts contradictoires, mais aussi en raison de leur recherche de puissance. Cette référence à la notion de puissance économique indique que, dans la configuration actuelle de l'économie mondiale, certaines unités économiques comme les firmes multinationales, entendent jouer un rôle dans la partie qui se joue à l'échelle mondiale. Dans le cas qui nous préoccupe ici, tendre vers la puissance est sous-tendu par des motifs industriels et concurrentiels ${ }^{9}$. En s'inspirant de la théorie des relations internationales, on peut donc dire que la puissance économique reflète la capacité d'une unité économique (État, firme multinationale) à s'imposer en tant qu'acteur clé sur le plan de l'économie mondiale. Il s'agit par conséquent d'un concept illustrant les rapports de force entre acteurs, ici industriels - la puissance économique pouvant

9. Jouer un rôle actif dans la mondialisation signifie que, si chaque acteur partage cette ambition, une logique conflictuelle en découle, et concerne autant les États que les firmes multinationales. Cette problématique s'inscrit dans les analyses produites par le courant de la « guerre économique ». Cf. Schmidt C. (1990). Penser la guerre, penser l'économie, Paris, Odile Jacob ; Bosserelle E. (2011). La guerre économique, forme moderne de la guerre ? Revue Française de SocioÉconomie, ${ }^{\circ}$ 8/2, p. 167-186. revêtir plusieurs formes (industrielle, démographique, monétaire...). Dans le cas des firmes multinationales de l'agroalimentaire, l'un des enseignements à retirer de la théorie des Firmes multinationales (FMN) est que l'implantation dans un pays étranger modifie la structure de marché d'un secteur, au profit des firmes les plus dynamiques (Smith, 1987) ${ }^{10}$.

L'échange international n'est pas neutre, et comme l'indiquait l'économiste français François Perroux : «Chaque État national ne met pas au service d'une clientèle anonyme les produits neutres de ses industries: toutes les marchandises battent pavillon; il n'est point de marchandise neutre ; il n'est pas d'industrie ni de commerce politiquement neutre. »(Perroux, 1958) ${ }^{11}$. Si le rôle de l'État est manifestement décisif dans le profil commercial d'une nation, de multiples recherches l'ont montré, à commencer par les analyses de Paul Krugman en matière de politique commerciale stratégique, il n'en demeure pas moins que ce sont des firmes qui définissent et renouvellent leur stratégie de conquête des marchés, en jouant sur leurs avantages comparatifs (Krugman, 1986) ${ }^{12}$.

L'exportation est en effet répertoriée comme une sortie de marchandises d'un pays A vers un pays B. À ce flux physique correspond en sens inverse un flux monétaire, correspondant au règlement que doit effectuer le pays importateur au pays exportateur. Il se trouve que les exportations (comme les importations), sont imputables à des comportements de firmes localisées dans des pays, et qui, au travers de leur stratégie, pensent l'exportation comme

10. Smith A. (1987). Strategic Investment, Multinational Corporations and Trade Policy. European Economic Review, $\mathrm{n}^{\circ}$ 31, p. 89-96.

11. Perroux F. (1958). La coexistence pacifique. Paris, Presses universitaires de France.

12. Krugman P. (ed.) (1986). Strategic Trade Policy and the New International Economics. Cambridge, Mass.; London, MIT Press. 


\section{FAITS ET CHIFFRES}

Graphique 3. Exportation mondiale de viande de volailles

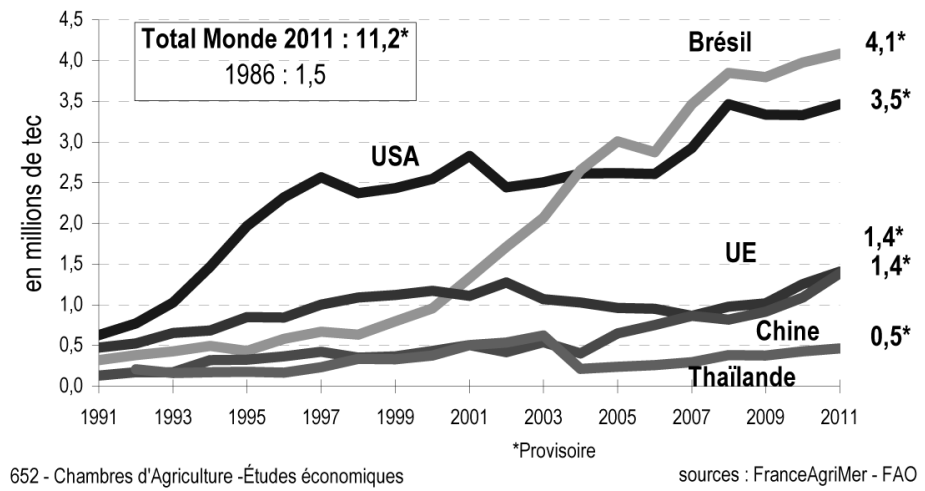

Graphique 4. Commerce extérieur du Brésil en agroalimentaire

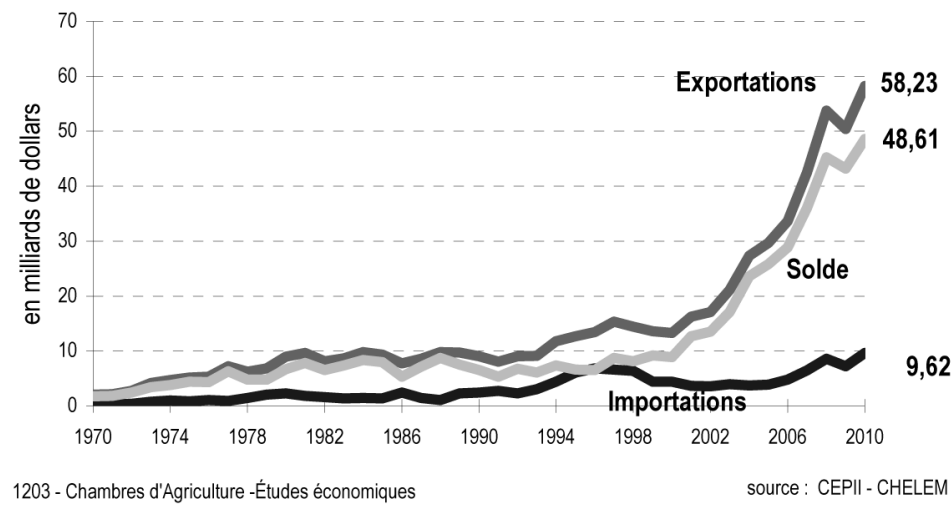

un vecteur de la conquête des marchés. On peut donc affiner les éléments qui ont été présentés ci-dessus, en indiquant qu'à l'échelle microéconomique, la progression des firmes brésiliennes de l'agroalimentaire à l'exportation, a conduit à une révision de la hiérarchie mondiale des entreprises de ce secteur. Antérieurement, les firmes qui dominaient les marchés étaient ou américaines ou européennes (Bunge, Cargill, Dreyfus...), avant que n'émergent des entreprises brésiliennes exportatrices de produits comme le poulet, le sucre ou le soja (SLC, Marfrig, JBS, Brasilagro...). Depuis plus d'une décennie, ces firmes se sont multinationalisées, et plus particulièrement celles qui transforment les viandes.

\section{L'affirmation des firmes multinationales brésiliennes des viandes}

Dans son rapport 2010 sur l'investissement dans le monde, la Conférence des Nations unies pour le commerce et le développement (CNUCED) indiquait qu'en Amérique latine, les FMN devenaient des acteurs de taille mondiale, participant ainsi activement à la structuration de l'économie 
FAITS ET CHIFFRES

Cécile FÈVRE, Thierry POUCH

Graphique 5. Exportations brésiliennes de graines oléagineuses vers l'Europe et la Chine

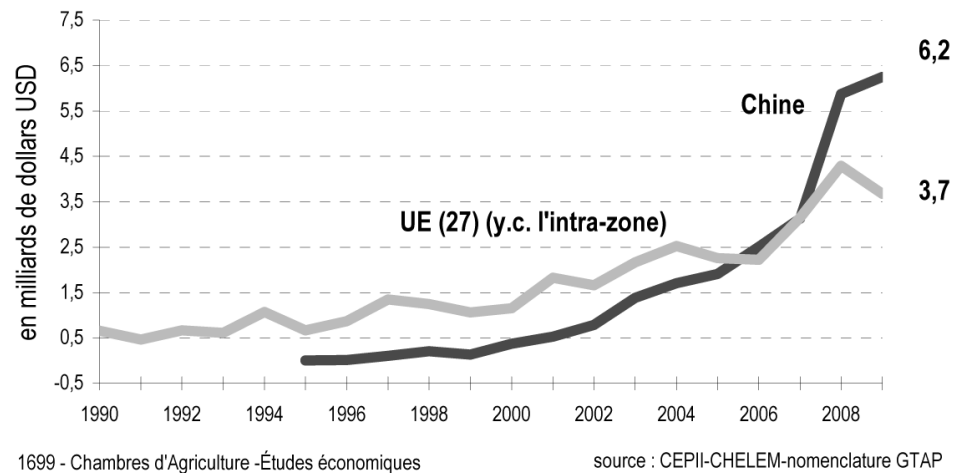

mondiale (CNUCED, 2010). En raison de sa forte croissance économique, le Brésil a largement contribué à élever la part de la Région sud-américaine dans les flux d'IDE sortants. Entre 1991 et 2000, les IDE sortants réalisés par les firmes brésiliennes s'élevaient en moyenne à quelque 1 milliard de dollars, pour passer à près de 12 milliards de dollars en moyenne entre 2003 et 2008. Cette croissance des IDE brésiliens fut davantage le fait d'opérations de fusions-acquisitions (Cross Bor$\operatorname{der} M \& A s)$ que de créations de filiales à l'étranger (Shimizu et al., 2004) ${ }^{13}$. Il s'agit par conséquent d'un indicateur intéressant au travers duquel on peut prendre la mesure $\mathrm{du}$ processus historique en cours, à savoir que le rythme des IDE émanant des pays émergents est supérieur à celui des IDE des économies industrialisées, signe que la hiérarchie des nations est en train d'évoluer depuis quinze ans. La progressive montée en puissance des firmes transnationales brésiliennes découle d'une politique économique instaurée durant la décennie 1990, et

13. Shimizu K. et al. (2004). Theoretical Foundations of Cross-Border Mergers and Acquisitions: A Review of Current Research and Recommendations for the Future. Journal of International Management, $\mathrm{n}^{\circ} 10$, p. 307-353. qui a consisté à pratiquer concomitamment une ouverture commerciale, une libéralisation de secteurs entiers de l'économie, débouchant non seulement sur une attractivité du territoire brésilien, mais aussi sur la formation de grands groupes multinationaux de l'agroalimentaire (Giordano, Santiso, 1999 ; Rastoin, Ghersi, 2010) ${ }^{14}$.

S'agissant du secteur de l'agroalimentaire, les FMN brésiliennes ont amplifié depuis le début des années 2000 le processus général de mondialisation des firmes et donc d'intégration de la production de produits alimentaires, tout en élevant un peu plus la part des sites de production brésiliens dans le PIB agroalimentaire mondial. L'interprétation que l'on peut livrer a trait à la stratégie du Brésil, tournée vers la conquête des marchés mondiaux, d'abord par la voie de l'exportation - il conviendrait d'ailleurs de montrer en quoi l'économie brésilienne a opéré une reconfiguration de ses avantages comparatifs durant

14. Giordano P., Santiso J. (1999). La course aux Amériques : les stratégies des investisseurs européens en Argentine et au Brésil. Les études du CERI, $\mathrm{n}^{\circ}$ 52, avril, p. 1-38 ; Rastoin J.-L., Ghersi G. (2010). Le système alimentaire mondial. Concepts et méthodes, analyses et dynamiques. Versailles, Quae. 
les années quatre-vingt-dix, en les centrant de plus en plus sur un secteur comme l'agroalimentaire, alors que cette économie était destinée à valoriser des secteurs comme l'automobile ou l'armement (Salama, 2010, 2011 $)^{15}$ - puis désormais par le truchement de l'internationalisation du capital agroalimentaire. Plusieurs grandes firmes se sont en effet positionnées d'abord sur le marché intérieur brésilien pour atteindre ensuite une taille d'envergure mondiale. C'est le cas en particulier de firmes de la viande comme JBS et Marfrig. Selon les données établies par Rabobank, JBS figure au premier rang des entreprises productrices de viande de volailles et bovine avec respectivement près de 4 et 7,7 millions de tonnes, tandis que Marfrig se classe au quatrième rang des firmes mondiales productrices de viande bovine, avec plus d'un million de tonnes, derrière sa concurrente brésilienne et derrière Cargill $^{16}$. En termes de chiffre d'affaires, $J B S$ dégageait 31 milliards de Reals en 2009, devançant ses concurrentes américaines et brésiliennes, Marfrig ne parvenant qu'à engendrer un peu moins de 10 milliards de chiffre d'affaires de ses activités. Il s'agit donc des premier et huitième groupes industriels mondiaux de la viande.

L'expansion de ces groupes brésiliens s'est accélérée à partir du début des années 2000. Si JBS constituait déjà, à la fin des années 1990, l'un des principaux abatteurs brésiliens de viande bovine (5000 têtes par jour), il multiplie ensuite les rachats d'entreprises brésiliennes et argentines, ces

15. Salama P. (2010). Brésil : bilan économique, succès et limites. Problèmes d'Amérique latine, $\mathrm{n}^{\circ}$ 78, automne, p. 47-63 ; Salama P. (2012). Chine-Brésil : industrialisation et désindustrialisation précoce. Économie appliquée, n 1, p. 33-69. Voir également Salama P. (2012), Les économies émergentes latino-américaines, entre cigales et fourmis, Paris, Armand Colin, coll. «U ». 16. Rabobank International (2010). Outlook for the global meat industry. dernières étant fragilisées par la grave crise économique des années 1990. La firme JBS a élargi de fait sa capacité d'abattage (par 4,5 ), accédant ainsi au rang de plus grande entreprise de transformation de produits carnés d'Amérique latine. Son introduction en bourse en 2007 lui permet de lever des fonds et de poursuivre sa stratégie d'expansion aux États-Unis, en Europe, et jusqu'en Australie, non seulement dans le secteur de la viande bovine mais également dans l'ensemble des autres productions carnées (porcs, volailles, ovins). Les firmes transnationales de l'agroalimentaire des pays du Nord (Nestlé, Danone, Unilever) avaient surtout investi dans les produits de grande consommation du type produits laitiers, biscuiterie, délaissant le secteur des viandes, dans lequel se sont, du coup, engouffrées les firmes brésiliennes, du fait d'une moindre intensité concurrentielle à l'échelle mondiale.

Aux États-Unis, JBS acquiert en 2007 Swift \& Compagny, troisième producteur américain de viande porcine, puis en 2009 Pilgrim's pride, plus grande entreprise de volaille américaine qui possède une partie de sa production au Mexique. Sa percée en Europe se concrétise par l'achat de $50 \%$ des parts de l'italien Crémonini dans Inalca $^{17}$ en 2007 puis, deux ans plus tard, d'une entreprise belge de transformation de viande bovine. Il entre également sur le marché russe en 2009 via le rachat d'une usine de transformation de hamburgers.

L'expansion de Marfrig, à partir de son ancrage brésilien, répond à cette même logique de croissance externe à travers de nombreuses acquisitions entre 2006 et

17. Depuis lors, $J B S$ a mis fin à son partenariat avec le Groupe Cremonini en lui revendant sa part de $50 \%$ dans la joint-venture avec Inalca mais a pris le contrôle de la société italienne Rigamonti Salumificio (un des leaders de la transformation de viandes fumées en Italie). Source : UBIFRANCE (2011). L'actualité des marchés au Brésil. www. ubifrance.fr. 
FAITS ET CHIFFRES

Cécile FĖVRE, Thierry POUCH

2008. Son implantation est forte dans les pays du MERCOSUR. En Uruguay, elle détient $30 \%$ de la production et $26 \%$ des exportations de viande bovine. En Argentine, elle contrôle les deux tiers du marché du steak haché après le rachat de la société Quickfood. Elle est également positionnée sur des marchés à forte valeur ajoutée dès lors qu'elle possède plusieurs marques liées à l'exportation de pièces de viande bovine de haute, voire très haute, qualité. En 2008, elle acquiert plusieurs entreprises du groupe américain OSI situées au Brésil (Braslo) et en Europe (Moy Park, Albert Van Zoonen, Kitchen Range). L'entreprise brésilienne Marfrig est tournée vers la production de viande de bœufs et de volailles à destination des marchés du Moyen-Orient et d'Asie pour fournir les chaînes de restauration rapide. Les acquisitions de sites industriels en Europe spécialisés dans la viande de volailles et de porcs ont propulsé Marfrig au rang de premier producteur de viande de volailles au Royaume-Uni. Ses produits sont destinés aux grandes chaînes de restauration rapide (du type MacDonald's, $K F C \ldots$ ) et de distribution (Tesco, Sainsbury's, Waitrose). La société Moy Park détient aujourd'hui une part importante du marché des morceaux de poulets et morceaux cuisinés (respectivement $30 \%$ et $60 \%$ au Royaume-Uni). Outre des usines en Angleterre, Marfrig possède également deux sites de production en France, situés dans le Nord-Pas-de-Calais, spécialisés dans la production pour des marques de distributeurs, la restauration commerciale et collective et des industriels (produits intermédiaires pour assemblages). Différentes acquisitions en 2009 et 2010 ont complété son portefeuille d'activités, en particulier celle de Sera, entreprise brésilienne de volailles rachetée à Cargill et Keystone Foods, deux sociétés américaines fournisseur de food-service ${ }^{18}$.

18. Dans 13 pays, 28000 restaurants, 13000 salariés, pour un chiffre d'affaires de 6,4 milliards de dollars. Source : ibid.
Cette dynamique de fusions-acquisitions impulsée par les grandes FMN brésiliennes est donc assez récente. Elle illustre le fait que, derrière la crise financière actuelle, se déroule une vaste restructuration du capital transnational, dont l'agroalimentaire constitue l'un des domaines saillants, débouchant non seulement sur des concentrations et implantations nouvelles d'unités de production dans le secteur de la transformation alimentaire, mais aussi sur une reconfiguration de la hiérarchie des FMN. Le couple concentration/implantation des unités de production indique que les FMN brésiliennes cherchent à articuler de façon optimale avantages microéconomiques propres à chaque firme et avantages comparatifs des nations et des territoires sur lesquels elles envisagent de s'implanter.

\section{Les déterminants des IDE agroalimentaires brésiliens}

Qu'est-ce qui motive une firme à préférer une implantation plutôt que l'exportation ? En quoi la comparaison des coûts fixes engendrés par cette localisation de la production dans un pays hôte et des coûts variables associés au transport et aux droits de douane débouche-t-elle sur un arbitrage en faveur de l'IDE ? La théorie de la multinationalisation des firmes suggère qu'une pluralité de facteurs explique l'IDE. Plusieurs auteurs ont analysé et évalué empiriquement les motifs conduisant à s'implanter sur un territoire (Dunning, 1993 ; Andreff, 2003 ; Navaretti, Venables, 2004 ; Rioux, 2012) ${ }^{19}$. Dans la mesure où

19. Dunning J. (1993). Multinational Enterprises and the Global Economy. Reading, MA: AddisonWesley Publishing ; Andreff W. (2003). Les multinationales globales. Paris, La Découverte, coll. « Repères »; Navaretti G., Venables A. J. (2004). Multinational Firms in the World Economy, Princeton: Princeton University Press ; Rioux M. (2012). Théories des firmes multinationales et des réseaux économiques transnationaux. Cahier de Recherche, CEIM-UQAM, mars, p. 1-37. 
les firmes brésiliennes s'implantent désormais dans des régions développées, à l'instar des IDE réalisés en Europe et en Océanie, voire aux États-Unis, les facteurs coûts de la main-d'œuvre et proximité des matières premières peuvent être écartés des déterminants ayant poussé ces firmes à pratiquer l'IDE. Il s'ensuit que le facteur de la localisation des unités de production brésiliennes du secteur des viandes relève davantage d'une stratégie de présence sur les marchés afin d'approvisionner au plus vite les consommateurs des pays d'accueil (la production sur place, en réduisant la distance, engendre annulation du coût de transport et baisse du coût marginal de production). De plus, les productions de viandes étant exposées aux accidents sanitaires, le fait d'être implanté sur plusieurs régions permet de minimiser le risque de crise sanitaire, toutes les zones n'étant pas simultanément touchées par les épizooties (fièvre aphteuse, grippe aviaire...). Le Brésil est en effet frappé régulièrement par des interdictions d'exporter vers le reste du monde du fait de la présence de foyers endémiques de maladies touchant les animaux.

Le cœur de la stratégie de ces groupes consiste donc d'abord à renforcer leur pouvoir de marché au Brésil et à internationaliser ensuite leurs activités au prix d'une campagne soutenue de fusions et d'acquisitions. Après avoir consolidé leur position à l'échelle du MERCOSUR en absorbant progressivement leurs principaux concurrents, ils ont multiplié les opérations d'achats pour s'implanter sur les marchés américains, australiens et européens. En outre, l'implantation en Australie et en Europe leur permet d'avoir une porte d'entrée sur les marchés du Moyen-Orient, d'Afrique du Nord, d'Europe de l'Est et d'Asie (voir carte 1). Ils se déploient dans les principaux pays producteurs de viande et sur les bassins de consommation les plus porteurs, minimisant ainsi certains coûts de transport.

La stratégie de localisation des firmes brésiliennes de l'agroalimentaire s'inscrit dans l'analyse, vérifiée empiriquement, produite par Brainard. Cet économiste a en effet entrepris d'expliquer pourquoi les IDE concernaient une part importante des pays industrialisés, non seulement comme investisseurs mais aussi et de plus en plus en tant que territoires d'accueil de ces IDE. L'arbitrage des firmes qui s'implantent s'effectue selon le critère concentrationproximité. Plus précisément, l'implantation à proximité des consommateurs est subordonnée à la réalisation d'économies d'échelle, à la minimisation des coûts d'implantation, à la croissance potentielle du marché intérieur (Brainard, 1993, 1997 ; Mouhoud, 2006) ${ }^{20}$.

Il a été rappelé plus haut que les productions brésiliennes de viande de volailles avaient supplanté assez largement les productions françaises sur les marchés du Moyen-Orient, allant même jusqu'à former un véritable " corridor alimentaire » entre elles et les consommateurs de ces pays cibles. L'évolution des IDE réalisés par des firmes comme Marfrig et $J B S$ a joué un rôle déterminant dans cette conquête des marchés du Moyen-Orient

20. Brainard S. L. (1993). A Simple Theory of Multinational Corporations and Trade with a Trade-Off Between Proximity and Concentration. NBER, Working Paper, $\mathrm{n}^{\circ} 4269$; Brainard S. L. (1997). An Empirical Assessment of the Proximity-Concentration Trade-Off Between Multinational Sales and Trade. American Economic Review, vol. 87, ${ }^{\circ} 4$, sept., p. 520-544 ; Mouhoud E. M (2006). Mondialisation et délocalisation des entreprises. Paris, La Découverte, coll. « Repères ». Voir également les analyses plus anciennes de Buckley P., Casson M. (1976). The Future of the Multinational Enterprise, Mac Millan ; Buckley P., Casson M. (1981). The Optimal Timing of Foreign Investment. Economic Journal, vol. 91, n 361, p. 75-87 ; Buckley P., Casson M. (1985). The Economic Theory of the Multinational Enterprise. London: Macmillan. 
FAITS ET CHIFFRES

au détriment d'acteurs jusque-là présents comme la France. Entre 1995 et 2006, les exportations françaises de volailles découpées se sont situées sur une tendance à la baisse, la remontée ne s'effectuant qu'en fin de période, sachant que ces exportations ont rechuté durant le premier semestre 2012 (graphiques 6 et 7). L'implantation en Europe a en effet offert l'opportunité de réduire les coûts de transports associés à la proximité géographique du marché moyen oriental. JBS et Marfrig font par conséquent partie de ces quelques grands groupes brésiliens qui ont acquis une envergure internationale et sont le moteur de l'extraversion de l'agro-industrie du Brésil, correspondant étroitement à la stratégie du Gouvernement brésilien définie il y a une quinzaine d'années. On peut noter par surcroît une adéquation de leur outil industriel avec les normes, standard et procédés industriels internationaux. Il découle que les IDE pratiqués par les firmes brésiliennes de la viande permettent d'économiser les coûts variables liés au transport et aux droits de douane.

La recherche de nouveaux débouchés et d'un élargissement de leurs parts de marché les conduisent au rachat et au contrôle d'outils de transformation sur les marchés les plus solvables, rémunérateurs ou en croissance. Il s'agit de s'appuyer sur des investissements dans les outils de transformation en Europe et aux États-Unis qui leur permettent d'accéder à des lignes de production et des procédés industriels. Ces groupes, qui ont débuté leurs activités dans le secteur de la viande bovine, sont aujourd'hui positionnés sur l'ensemble de l'offre en produits carnés (viandes bovine, porcine, ovine et de volaille). Ils poursuivent un objectif de diversification de leur offre en produits transformés : produits découpés, cuisinés, destinés essentiellement aux GMS et aux chaînes de restauration rapide. Ils s'inscrivent dans ce processus d'internationalisation/ homogénéisation des modes de consommation alimentaire. De plus, ces groupes développent des unités d'engraissement (États-Unis, Argentine, Brésil, Australie), signe d'une stratégie ciblant le contrôle de l'ensemble de la chaîne de valeur (engraissement, abattage, transformation et commercialisation). On assiste donc à une dynamique d'intégration des filières animales portée par les firmes multinationales brésiliennes : fonctionnement sur le mode hiérarchique pour réduire les coûts de transaction et optimiser la valorisation de la viande en jouant sur les demandes spécifiques et complémentaires des pays importateurs ; hiérarchie également pour jouer sur les différentiels de coûts de production de la matière première entre des zones géographiques tributaires de l'évolution des taux de change, des coûts de transport...

Les pouvoirs publics brésiliens ont joué un rôle important dans le développement des groupes agroalimentaires. Ils ont en effet ouvert leur capital à des investisseurs privés et à des institutions bancaires, débouchant sur une interpénétration capital industriel/capital bancaire. Ainsi, la Banque de développement du Brésil (BNDES) détiendrait $20 \%$ de JBS et $14 \%$ de Marfrig. Elle aurait apporté depuis 2008 plus de 4 milliards de dollars pour financer les investissements des grandes entreprises brésiliennes de la viande. D'autres sources indiquent que l'achat de Pilgrim's pride aux États-Unis s'est concrétisé grâce à un prêt avantageux concédé par la BNDES. Il est par conséquent vain d'opposer État-nation et firmes multinationales. Ces groupes ont bénéficié par ailleurs du développement des marchés financiers, c'est-à-dire de l'intérêt des investisseurs pour les activités agro-industrielles. Ils ont ainsi réalisé leur introduction en bourse pour financer l'internationalisation de leurs activités industrielles.

L'internationalisation des échanges de produits carnés s'accompagne donc de l'émergence de multinationales de la 


\section{FAITS ET CHIFFRES}

Graphique 6. Exportations françaises de volailles vers le Moyen-Orient

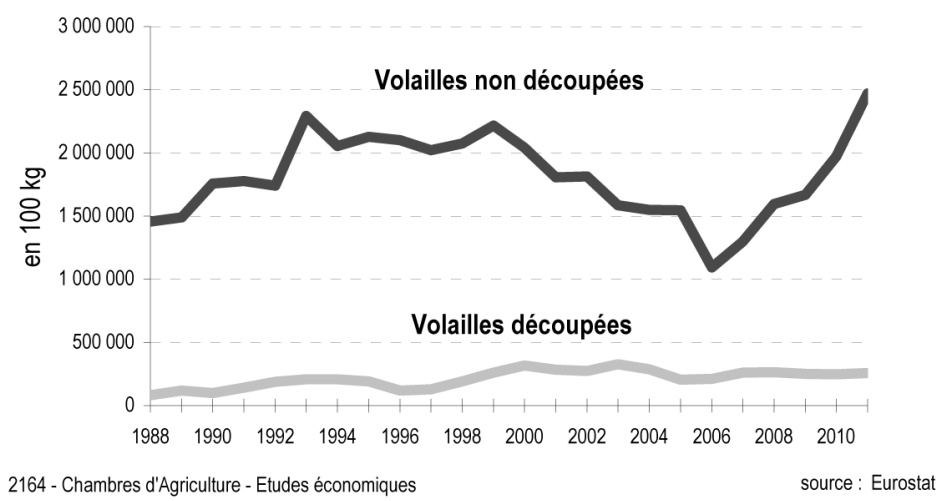

viande qui contrôlent une part croissante du marché. Ces acteurs s'orientent vers une production de masse, standardisée, qui fait ensuite l'objet d'un écoulement approvisionnement des grandes chaînes de restauration rapide et de distribution. Il faut y voir la conjonction d'un modèle de production et d'échange avec un modèle de consommation globalisée. Une forte restructuration est à l'œuvre parmi les groupes industriels de l'abattage, de la découpe et de la transformation. Quelles en sont les incidences sur les autres maillons des filières ? Cette dynamique agroindustrielle fait craindre une exacerbation de la concurrence envers les productions animales européennes. Le risque réside dans la capacité de prédation qui anime les FMN brésiliennes et qui pourraient ainsi se lancer à la conquête, par acquisitions

Graphique 7. Exportations brésiliennes de viande de volailles et bovine à destination du Proche et Moyen-Orient

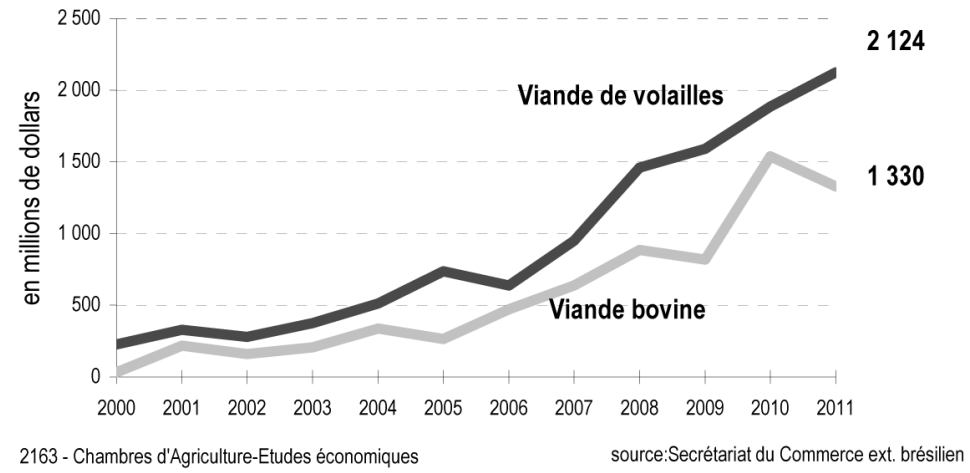


FAITS ET CHIFFRES

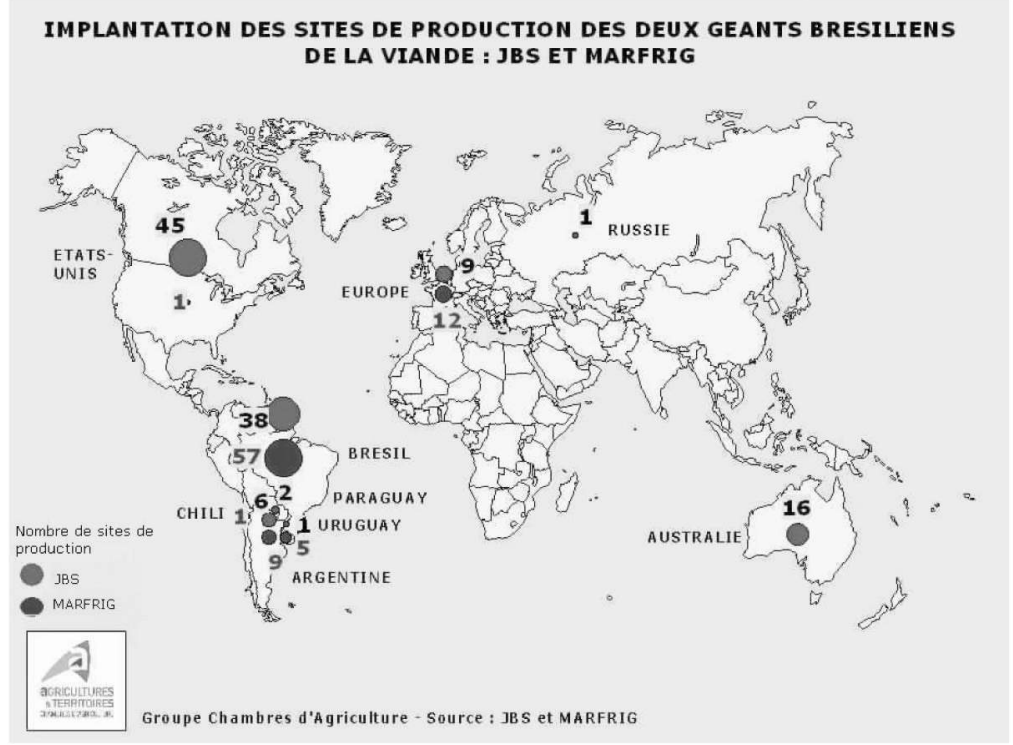

et/ou fusions, de leurs concurrents européens $^{21}$. En fragilisant ces derniers, la crise économique actuelle expose les unités de production européennes aux appétits de puissance agro-industrielle brésiliens.

$$
\begin{gathered}
* \\
* *
\end{gathered}
$$

L'enseignement global que l'on peut retirer de cette dynamique des groupes multinationaux brésiliens du secteur des viandes tient à ce qu'ils participent, dans la globalisation des industries de la transformation alimentaire, de la configuration de l'offre et de la demande mondiales de viandes. S'inscrivant dans cette perspective désormais bien établie d'une progression de la demande mondiale de produits carnés, émanant en particulier des économies dites émergentes, les FMN brésiliennes des

21. Sur la notion de prédation, très répandue en économie industrielle, en particulier dans le domaine des prix, se reporter à Tirole J. (1995). Théorie de l'organisation industrielle. Tome II, Paris, Economica. viandes se positionnent comme des maillons fondamentaux de l'économie agroalimentaire mondiale.

Si l'on adopte une problématique davantage centrée sur la rivalité des nations et des firmes productrices de biens alimentaires, du coup considérées comme des acteurs clés de la géoéconomie des productions de viandes, la géoéconomie pouvant se définir comme un espace de rivalités économiques et commerciales afin de conquérir ou préserver des parts de marché, l'émergence de géants agroalimentaires brésiliens invite à réexaminer les déterminants de l'échange international. L'affirmation des FMN brésiliennes de la viande confirme bien, de ce point de vue, que l'économie mondiale agricole et alimentaire voit son centre de gravité basculer du côté de l'Amérique latine (Hugon, Marques Pereira, 2011)22.

22. Hugon P., Marques Pereira J. (2011). Économie politique tricontinentale : les nouveaux paradigmes Suds-Suds, introduction. Revue TiersMonde, $\mathrm{n}^{\circ}$ 208, octobre-décembre, p. 7-27. 


\section{FAITS ET CHIFFRES}

Toutes les branches de l'agriculture et de l'agroalimentaire ne sont pas pour autant également performantes sur les marchés mondiaux. Plus généralement, n'est-ce pas aussi le signe annonciateur que, dans le domaine de l'agroalimentaire, les firmes des pays du Sud progressent au détriment de leurs concurrentes du Nord ? Finalement, rappelons que, tous secteurs confondus, le nombre de FMN du Sud est passé de 2800 à 18000 entre 1990 et le début des années 2000 (Ruet, 2007) ${ }^{23}$.

Les auteurs tiennent à remercier Dominique Redor (Université de Paris-Est Marne-la-Vallée), ainsi que les deux rapporteurs anonymes, pour leurs critiques et remarques constructives ayant permis d'améliorer une première version de ce texte. Les erreurs qui subsisteraient restent $d u$ seul ressort des auteurs.
23. Ruet J. (2007). Émergence des firmes multinationales « du Sud » et lecture du capitalisme. Annuaire Français des Relations Internationales, IFRI, p. 808-825 